\title{
Estudio de liderazgo en una carrera de educación superior, en una universidad regional
}

\author{
[Study of leadership in a career of tertiary education in a regional university] \\ Alberto Martínez Quezada*, Gabriel Díaz Flores, Claudia Araya Medina \\ Facultad de Ingeniería y Arquitectura, Campus Playa Brava, Universidad Arturo Prat, Av. Arturo Prat 2120, Iquique, Chile. \\ *e-mail de contacto: albmarti@unap.cl; alberto.martinez@unap.cl
}

\begin{abstract}
Resumen
El objetivo del presente trabajo es determinar estilo de liderazgo presente en la dirección de carrera de Ingeniería Civil Industrial, de la Universidad Arturo Prat de Iquique. Para el levantamiento de información, se aplicó el Cuestionario Multifactorial de Liderazgo MLQ 5X, adaptado a la realidad organizacional chilena, a los académicos y al director de carrera. El estudio permitió determinar que, de los estilos de liderazgos evaluados (Liderazgo transformacional, Liderazgo transaccional y Laissez faire), el líder estudiado presenta características del Liderazgo transformacional y transaccional, con una diferencia cercana al $9 \%$, siendo mayor el primero, logrando aplicar ambos estilos de liderazgo de acuerdo al contexto, en conjunto con otras variables como la efectividad y la satisfacción evaluada por los clasificadores. Este resultado es respaldado por el estudio y análisis, del "Modelo de las nueve dimensiones de rango total".
\end{abstract}

Palabras Clave: Liderazgo; Liderazgo Transformacional; Liderazgo Transaccional; MLQ

\begin{abstract}
The objective of this study is to determine the leadership style of the Director of Industrial Engineering Programme of Arturo Prat University, located in Iquique, Chile. For information-collecting process, a Chilean organizational reality adapted Multifactor Leadership Questionnaire MLQ 5X was applied to academics and Director. The study determines that of the evaluated leadership styles (Transformational Leadership, Transactional leadership and Laissez faire), the leader has characteristics of transformational and transactional leadership with a difference of about $9 \%$, being the first of them higher, achieving both leadership styles according to the context, along with other variables such as effectiveness and satisfaction, evaluated by the classifiers. This result is supported by the study and analysis of "Model of the nine dimensions of full range leadership".
\end{abstract}

Keywords: Leadership; Transformational Leadership; Transactional Leadership; MLQ 


\section{INTRODUCCIÓN}

Dada la importancia del liderazgo para el éxito o fracaso de las organizaciones, a lo largo del tiempo se han planteado muchas interrogantes que giran en torno a la idea del "¿Qué hace a un gran líder?". Los intentos por responder esta pregunta se remontan a los primeros debates sobre el concepto de liderazgo. Sin embargo, es en este último medio siglo que el liderazgo se ha convertido en una importante área de investigación académica (Judge y Bono, 2000).

La carrera de Ingeniería Civil Industrial de la Universidad Arturo Prat de Iquique, se ha impartido durante 31 años hasta la fecha. El Consejo Nacional de Acreditación de Chile (CNA) le ha otorgado 5 años de acreditación de un número máximo de 7 años para las carreras de instituciones universitarias. En tanto, solo tres carreras de la universidad cuentan con la misma cantidad de años de acreditación y la universidad posee una acreditación por 3 años definidos por la CNA. Ingeniería Civil Industrial es la carrera que recibe la mayor cantidad de alumnos nuevos completando sus cupos al segundo día de postulación, además de poseer los mayores puntajes PSU de ingreso a primer año de universidad (CNA-Chile, 2016).

Es posible reconocer, según estadísticas de la carrera, un número significativo de egresados posicionados en cargos ejecutivos en importantes industrias de la región y del país como la minería, bancos, pesqueras, comercio y Gobierno. En ese aspecto, se reconoce la importancia del liderazgo como tema en desarrollo constante a lo largo del período universitario, es más, el último semestre de la carrera, se dicta la asignatura de "taller de creatividad y liderazgo" donde los alumnos analizan y se familiarizan con cada uno de los tipos de liderazgo y como inciden en diferentes organizaciones. La asignatura culmina con la realización de una investigación acerca del tipo de liderazgo que se ejerce en alguna organización de una importante industria en el extranjero. Con los resultados obtenidos los alumnos deben construir una publicación científica que plasme la identificación del tipo de liderazgo presente en la organización en estudio.

Desde este punto de vista, se reconoce la importancia del liderazgo en esta profesión y por ende, es factible un análisis al tipo de liderazgo que se ejerce en la Dirección de
Carrera y como éste influye en sus seguidores (UNAP, 2016).

Durante casi tres décadas la teoría del liderazgo ha desarrollado un avance significativo, estructurando en cuerpo y forma de los estilos de liderazgo transformacional y transaccional (Judge y Piccolo, 2004). En 1978, Burns propuso estos conceptos de liderazgo (Burns, 1978), en los que Bernard Bass se inspiró y comenzó a desarrollar posteriormente (Judge y Bono, 2000). Burns pudo diferenciar dos estilos de liderazgo, el liderazgo transformacional del transaccional (Lowe et al., 1996), y basado en esa conceptualización, Bass desarrolló su propia teoría del liderazgo con varias modificaciones, ya que éste no consideraba, como Burns, que los liderazgos transformacional y transaccional representaban extremos opuestos de un mismo objeto, sino conceptos separados, y que los mejores lideres eran aquellos que transformacionales y transaccionales a la vez (Judge y Piccolo, 2004).

El líder transformacional inspira a sus seguidores a identificarse con una visión más allá de los intereses propios por el bien del equipo (Burns, 1978; Den Hartog et al., 1997). El liderazgo transformacional mantiene una dinámica donde existe una fuerte identificación personal con el líder, donde se comparte una visión de futuro (Den Hartog et al., 1997). Los líderes transformacionales cambian la cultura, a través de la comprensión de ésta y luego la realinean con una nueva visión que sienta sus bases en conjeturas, valores y normas compartidas (Bass, 1985). Estos líderes ven nuevas formas de trabajar, nuevas oportunidades haciendo frente al riesgo, y prefieren respuestas efectivas a respuestas eficientes (Lowe et al., 1996). Ellos no se limitan a reaccionar ante las perturbaciones del entorno, sino que intentar crear estas perturbaciones y darles forma (Avolio and Bass, 1988). Cuatro dimensiones caracterizan a los líderes transformacionales. Estos factores incluyen la Influencia Idealizada, Motivación Inspiradora, Estimulación Intelectual y Consideración Individualizada (Bass y Avolio, 1993, Judge y Piccolo, 2004). Los líderes transaccionales trabajan con la cultura de su organización, siguiendo reglas, sus acciones y decisiones están enmarcadas en base a las normas operativas y procedimientos existentes. Estos líderes se caracterizan por la 
Recompensa contingente, la Administración por excepción Pasiva y Administración por excepción Activa. En esencia, existe un intercambio entre el líder y sus seguidores, proporcionando beneficios para éstos sí actúan según los deseos del líder, una relación de costo-beneficio entre ambos (Bass y Avolio, 1993, Burns, 1978, Den Hartog et al., 1997).

Las dimensiones de los liderazgos transformacional y transaccional contenidas en la Teoría del Liderazgo de Rango Total, son descritas en la Tabla 1.

Estudios realizados demuestran que el liderazgo transformacional se encuentra altamente relacionado con la efectividad del líder (Lowe et al., 1996). Además existe evidencia que afirma la validez del liderazgo transformacional en diversas culturas utilizando una variedad de métodos (Bass, 1997). Los líderes transformacionales pueden utilizar estrategias transaccionales cuando es apropiado, pero solo también, y con mayor frecuencia que lo anterior, hacen uso de elementos simbólicos en un intento de incrementar el esfuerzo y motivación de sus seguidores (Lowe et al., 1996).

Una de las diferencias claves entre el liderazgo transformacional y transaccional es que, mientras el líder transaccional motiva a sus seguidores a alcanzar las expectativas planteadas, el líder transformacional inspira a sus seguidores a obtener logros más allá de lo originalmente planificado (Den Hartog et al., 1997). No obstante, la mayoría de los lideres no es transformacional o transaccional, más bien presenta un comportamiento en el que conviven ambos estilos (Eagly and Johannesen-Schmidt, 2007).

El Cuestionario Multifactorial de Liderazgo (MLQ) se ha situado en la historia como el primer instrumento que mide elementos del liderazgo de forma cuantitativa. Inicialmente se evaluaron un conjunto de 142 ítems obtenidos por una investigación mixta a través de revisiones bibliográficas y la aplicación de una encuesta abierta a 70 altos ejecutivos de organizaciones reconocidas, donde describían atributos de ambos tipos de líderes (Lowe et al., 1996). El análisis factorial indicó cinco escalas de confiabilidad aceptable. Finalmente fueron reanalizados 73 ítems proporcionando resultados similares (Hater and Bass, 1988).

El perfil óptimo se basa en la aplicación de un liderazgo que presenta gran efectividad en el desempeño de los seguidores, con la ejecución de manera principal, más no exclusiva, de Liderazgo Transformacional. Se debe reconocer la importancia del liderazgo transaccional, ya que este estilo de liderazgo es eficaz bajo algunas culturas organizaciones. No obstante, autores indican que el Liderazgo Transformacional aumenta los efectos del Liderazgo Transaccional. Por lo que se considera que "los mejores lideres típicamente exhiben ambos liderazgo transformacional y transaccional" (Avolio et al., 1999).

El liderazgo educativo influye en los demás con el fin de realizar actividades que podrían mejorar el aprendizaje de los estudiantes, estableciendo metas comunes para la comunidad educativa. Estudios identifican al liderazgo como una de las variables más importantes, explicando varianza entre $5 \%$ y $7 \%$ entre instituciones educativas, siendo la segunda variable de mayor importancia después del contexto socioeconómico. Incluso de mayor relevancia en contexto de alta vulnerabilidad. El liderazgo no afecta de forma directa el aprendizaje de los alumnos, sino que fluye a través del trabajo de los docentes; interviniendo de forma directa la motivación, habilidades y satisfacción laboral entre todos los actores de la institución educativa (Horn and Marfán, 2010).

(Thieme, 2005) evidenció en su estudio que de las dimensiones del liderazgo de rango total, la que presenta una mayor incidencia es la influencia idealizada del líder, que es altamente respetado, los estudiantes y docentes confían en él y reconocen sus altos valores morales y éticos. Seguida por la motivación inspiradora y la recompensa contingente.

De igual forma, no es raro encontrar "gerentes educacionales" que han escalado en puestos gerenciales con baja o inexistente preparación directiva o gerencial o conocimientos de gestión organizacional, requiriendo tiempo para poder adaptarse a su nuevo trabajo (Rubino, 2007).

A continuación se presentará un estudio que permite identificar el estilo de liderazgo presente en la Dirección de Carrera de Ingeniería Civil Industrial. El liderazgo ha sido estudiado desde varias perspectivas según cada escuela en particular, dentro de los modelos desarrollados este estudio utilizará las bases que componen el Liderazgo de rango total, el cual encasilla a los líderes como 
Transaccionales, Transformacionales o Laissezfaire. El modelo del liderazgo de rango total está estructurado en base a 9 dimensiones, propuestas por Bass y Avolio (1993). Las características particulares de esta carrera dentro de la institución determinan la necesidad de investigar si los factores de liderazgo pueden incidir en la realidad de esta Escuela de Ingeniería Civil Industrial.

Igualmente se presentará la base teórica del presente estudio, que fue el resultado de la selección de otros artículos, trabajos y literatura acerca del liderazgo en diversos ámbitos.

Tabla 1: Dimensiones de liderazgo de rango total y respectiva caracterización.

\begin{tabular}{|c|c|c|c|}
\hline Variables Directas & Características del líder & Frase & Referencias \\
\hline $\begin{array}{l}\text { Influencia } \\
\text { Idealizada }\end{array}$ & $\begin{array}{l}\text { - Personifica un modelo carismático. } \\
\text { - Ofrece a sus seguidores un sentido del } \\
\text { propósito, una imagen, un modelo a } \\
\text { seguir. } \\
\text { - Apelan al nivel emocional haciendo que los } \\
\text { seguidores se identifiquen con él. }\end{array}$ & $\begin{array}{c}\text { "Si cree que es } \\
\text { correcto, } \\
\text { entonces..." }\end{array}$ & $\begin{array}{l}\text { (Judge and Bono, } \\
\text { 2000, Judge and } \\
\text { Piccolo, 2004, Den } \\
\text { Hartog et al., 1997, } \\
\text { Avolio et al., 1999, } \\
\text { Riaga and Torres, } \\
\text { 2006) }\end{array}$ \\
\hline $\begin{array}{l}\text { Motivación } \\
\text { Inspiradora }\end{array}$ & $\begin{array}{l}\text { - Articula una visión clara, atractiva y } \\
\text { estimulante para los seguidores. } \\
\text { - Plantea de forma optimista los logros y } \\
\text { metas esperadas, proporcionando un } \\
\text { significado a las tareas entregadas. }\end{array}$ & $\begin{array}{l}\text { "Espero su mejor } \\
\text { esfuerzo..." }\end{array}$ & $\begin{array}{l}\text { (Judge and Bono, } \\
\text { 2000, Judge and } \\
\text { Piccolo, 2004, Bass, } \\
\text { 1998, Den Hartog et } \\
\text { al., 1997, Riaga and } \\
\text { Torres, 2006) }\end{array}$ \\
\hline $\begin{array}{l}\text { Estimulación } \\
\text { Intelectual }\end{array}$ & $\begin{array}{l}\text { - Estimula la creatividad de los seguidores a } \\
\text { través del cuestionamiento de lo } \\
\text { establecido y el planteamiento de nuevos } \\
\text { desafíos. } \\
\text { - Solicita colaboración e ideas a sus } \\
\text { seguidores, toma riesgo, retando el status } \\
\text { quo. }\end{array}$ & $\begin{array}{c}\text { "¿Qué piensa } \\
\text { hacer frente } \\
\text { a...?" }\end{array}$ & $\begin{array}{l}\text { (Judge and Bono, } \\
\text { 2000, Judge and } \\
\text { Piccolo, 2004, Lowe et } \\
\text { al., 1996, Den Hartog } \\
\text { et al., 1997, Bass, } \\
\text { 1985, Avolio et al., } \\
\text { 1999, Riaga and } \\
\text { Torres, 2006) } \\
\end{array}$ \\
\hline $\begin{array}{l}\text { Consideración } \\
\text { Individual }\end{array}$ & $\begin{array}{l}\text { - Comprende a cada seguidor, atiende y } \\
\text { entrega soporte a las necesidades } \\
\text { individuales de los seguidores. } \\
\text { - Desarrolla un trabajo continuo junto al } \\
\text { seguidor, proporcionando } \\
\text { retroalimentación constante y escuchando } \\
\text { sus necesidades e inquietudes, para } \\
\text { alcanzar todo su potencial. }\end{array}$ & $\begin{array}{l}\text { "Me interesa que } \\
\text { usted se } \\
\text { desarrolle } \\
\text { profesionalmente } \\
\text { y haga carrera } \\
\text { en esta } \\
\text { organización..." }\end{array}$ & $\begin{array}{l}\text { (Bass, 1995, Avolio et } \\
\text { al., 1999, Den Hartog } \\
\text { et al., 1997, Judge } \\
\text { and Piccolo, 2004, } \\
\text { Riaga and Torres, } \\
\text { 2006) }\end{array}$ \\
\hline $\begin{array}{l}\text { Reconocimiento } \\
\text { Contingente }\end{array}$ & $\begin{array}{l}\text { - Provee transacciones constructivas o } \\
\text { intercambio de recursos, generalmente } \\
\text { económicos, por el apoyo del seguidor. } \\
\text { - Los seguidores pueden participar en este } \\
\text { intercambio sin estar estrechamente } \\
\text { comprometidos con los objetivos. }\end{array}$ & $\begin{array}{l}\text { "Si se hace como } \\
\text { se espera..." }\end{array}$ & $\begin{array}{l}\text { (Den Hartog et al., } \\
\text { 1997, Judge and } \\
\text { Piccolo, 2004, Judge } \\
\text { and Bono, 2000, } \\
\text { Avolio et al., 1999, } \\
\text { Riaga and Torres, } \\
\text { 2006) } \\
\end{array}$ \\
\hline $\begin{array}{l}\text { Administración por } \\
\text { Excepción Activa }\end{array}$ & $\begin{array}{l}\text { - Supervisa activamente el rendimiento para } \\
\text { poder anticipar desviaciones de lo } \\
\text { planificado. } \\
\text { - Cuando ocurren irregularidades, plantea } \\
\text { acciones correctivas para mantener los } \\
\text { niveles de rendimiento esperado. }\end{array}$ & $\begin{array}{l}\text { "Si observo que } \\
\text { no se está } \\
\text { haciendo algo..." }\end{array}$ & $\begin{array}{l}\text { (Judge and Bono, } \\
\text { 2000, Avolio et al., } \\
\text { 1999, Den Hartog et } \\
\text { al., 1997, Riaga and } \\
\text { Torres, 2006) }\end{array}$ \\
\hline $\begin{array}{l}\text { Administración por } \\
\text { Excepción Pasiva }\end{array}$ & $\begin{array}{ll}\text { - } & \text { Supone su reacción e intervención } \\
\text { únicamente cuando los problemas se } \\
\text { tornan graves. } \\
\text { - } \quad \text { Evita el cambio del status quo. }\end{array}$ & $\begin{array}{l}\text { "Si no se llevara } \\
\text { a cabo..." }\end{array}$ & $\begin{array}{l}\text { (Judge and Bono, } \\
\text { 2000, Avolio et al., } \\
\text { 1999, Riaga and } \\
\text { Torres, 2006) }\end{array}$ \\
\hline
\end{tabular}




\section{Laissez-faire \\ MATERIAL Y MÉTODOS Metodología}

Evita tomar cualquier decisión en absoluto

y transfiere la responsabilidad a sus seguidores.

- Ofrece poco en términos de dirección y soporte.
"Es indiferente si (Avolio et al., 1999,
lo hace o no lo Riaga and Torres, hace..." 2006)

Se realizó el levantamiento de información mediante la aplicación del instrumento MLQ 5X, adaptado a un formulario web forma seguidor, a los docentes de planta de Ingeniería Civil Industrial. Igualmente se aplicará el mismo instrumento pero en su forma líder al director de Carrera, quien cumple roles de dirección, teniendo personal a cargo, por lo que la evaluación del estilo de liderazgo es necesaria para implementar estilos de liderazgo adhoc a la institución.

\section{Diseño del Instrumento}

Para poder realizar la medición sobre el estilo de liderazgo presentado El Director de Carrera Ingeniería Civil Industrial de la Universidad Arturo Prat (UNAP), el instrumento utilizado fue un formulario web la cual contaba con 82 preguntas, estas estaban basadas en el Cuestionario de Liderazgo Multifactorial 5X versión corta, forma seguidor para los docentes y forma líder para el Director de Carrera, instrumento adaptado para el contexto de organizaciones chilenas (Vega-Villa y ZavalaVillalón, 2004).

\section{Instrumento de evaluación}

Este cuestionario constó con la validación de Humberto Gómez Ortiz, Psicólogo Organizacional y académico de la carrera de Psicología de la Universidad Arturo Prat, quien realizó una evaluación de la encuesta y determinó que existía concordancia entre las preguntas y los objetivos, también observó que las preguntas se encontraban bien redactadas y que éstas eran coherentes.

\section{Técnica de Análisis}

Luego de la recopilación de los datos necesarios y relacionados con el objetivo de esta investigación, la información es sometida a un análisis de carácter cuantitativo que consiste básicamente en tabular, organizar, categorizar e identificar los rasgos útiles para la posterior comparación.

Se inicia la selección de las categorías relacionadas a los estilos de liderazgos planteados anteriormente en la investigación: Transformacional, Transaccional y Laissez-
Faire. Posteriormente cada categoría se dividió en 9 subcategorías entregadas por los distintos autores estudiados, esto permitió organizar la información recopilada y obtener resultados de acuerdo a los objetivos propuestos. Luego de esto se realizó una breve descripción de cada sub-categoría resaltando las características esenciales de cada una de ella facilitando la clasificación de la información. En último lugar se ejecutó la comparación entre la información obtenida en base al cuestionario y la teoría previamente analizada clasificando cada frase en las distintas sub-categorías mediante este último paso se logra identificar el tipo de liderazgo o los tipos de liderazgos que presenta el Director de Carrera de Ingeniería Civil Industrial.

\section{RESULTADOS}

Los resultados obtenidos del Cuestionario multifactorial de liderazgo (MLQ) aplicados a los académicos jornada completa adscritos a la carrera de Ingeniería Civil Industrial presentan una desviación estándar de 0,81 promedio. Los resultados de la aplicación de este instrumento se encuentran tabulados en la Tabla 2.

\section{Liderazgo Transformacional}

Los ítems dirigidos a determinar el liderazgo transformacional presentan una evaluación positiva, definida por la respuesta "A menudo", posición 3 en la escala de Likert (0-4). Destacando las dimensiones de Estimulación Intelectual y Consideración Individual, alcanzando porcentajes de $71,9 \%$ y $75,0 \%$ respectivamente.

Los resultados más homogéneos se encuentran en la dimensión Influencia Idealizada Atribuida, indicados en los siguientes ítems

El ítem 10, "Me siento orgulloso/a de estar asociado con él", presenta la menor desviación estándar $(0,4629)$, y sus respuestas son "A menudo" o "Frecuentemente, sino Siempre".

El ítem 58, "Para mi él es un modelo a seguir", presenta la menor desviación estándar $(0,4629)$, y sus respuestas son "A veces" o "A menudo".

Además, una de la respuesta más alta evaluada pertenece a la dimensión Estimulación 
Intelectual. El ítem 73, "Me estimula a expresar mis ideas y opiniones sobre el método de trabajo", es una de las preguntas mejor evaluadas $(3,33)$ y con baja dispersión $(0,5164)$.

De la dimensión Consideración Individualizada, el ítem 19, "Por el bienestar del grupo es capaz de ir más allá de sus intereses", presenta la desviación estándar más alta $(1,3887)$ y está evaluada con un puntaje 3 de 4 , lo que la encasilla en "A menudo".

\section{Liderazgo Transaccional}

Los ítems dirigidos a determinar el liderazgo transaccional presentan evaluaciones entre "A veces" y "A menudo", valores 2 y 3 en la escala de Likert. Obteniendo un $75 \%$ en la dimensión Reconocimiento Contingente y $45,8 \%$ en Administración por excepción Pasiva, siendo esta ultima una de las únicas en contar con evaluación "A veces" (2)

La dimensión Administración por Excepción Pasiva cuenta con una de las respuestas más variadas. El ítem 12, "Se decide a actuar sólo cuando las cosas funcionan mal.", es uno de los ítems con desviación más alta $(1,3024)$.

\section{Laissez-faire o No Liderazgo}

Los ítems dirigidos a evaluar la dimensión Laissez-faire se encuentra evaluada como "A veces", valor 2 en la escala de Likert, alcanzando un 40,6\%, siendo uno de los ítems con evaluación más baja. Ejemplo de esto son los siguientes ítems:

El ítem 7, "Suele estar ausente cuando surgen problemas importantes", presenta la evaluación más baja $(0,625)$, encontrándose encasillada por "Rara vez", y presenta una desviación estándar de 0,5175.

El ítem 5, "Le cuesta involucrarme cuando surge alguna situación relevante", está evaluada como 1 y encasillada como "Rara vez". Este ítem presenta una desviación estándar de 0,7559.

\section{Elementos complementarios}

Las dimensiones Efectividad, Esfuerzo extra y Satisfacción se encuentran evaluadas por un conjunto de 17 ítems, la evaluación individual de cada ítem se encuentran evaluadas como "A menudo", valor 3 en la escala de Likert y entrega porcentajes de $75,0 \%, 70,8 \%$ y $75,0 \%$ respectivamente. El ítem 70, "Encuentro satisfacción al trabajar con él", presenta la pregunta con mayor puntaje bruto, alcanzando 3,375 de un total de 4, encasillándola dentro de "A menudo".

En base a los resultados obtenidos, se construyó el perfil de liderazgo del director de carrera, véase Figura 1. Este presenta una mayor densidad en el cuadrante superior derecho, que indica características de mayor actividad y eficacia.

Tabla 2: Distribución de respuestas obtenidas de la aplicación del MLQ a docentes de planta asociados a la carrera de Ingeniería Civil Industrial, 2016.

\begin{tabular}{|c|c|c|c|}
\hline $\begin{array}{c}\text { Variable de Orden } \\
\text { Superior }\end{array}$ & Variables Directas & Ítems & Puntaje real/Puntaje ideal \\
\hline \multirow{5}{*}{$\begin{array}{l}\text { Liderazgo } \\
\text { Transformacional }\end{array}$} & Influencia Idealizada Atribuida & 7 & $19 / 24$ \\
\hline & Influencia Idealizada Conducta & 8 & $23 / 32$ \\
\hline & Motivación Inspiradora & 8 & $23 / 32$ \\
\hline & Estimulación Intelectual & 8 & $23 / 32$ \\
\hline & Consideración Individual & 8 & $24 / 32$ \\
\hline \multirow{3}{*}{$\begin{array}{l}\text { Liderazgo } \\
\text { Transaccional }\end{array}$} & Refuerzo Contingente & 6 & $18 / 26$ \\
\hline & Administración por Excepción Activa & 6 & $15 / 20$ \\
\hline & Administración por Excepción Pasiva & 6 & $11 / 24$ \\
\hline No Liderazgo & Laissez-faire & 8 & $13 / 32$ \\
\hline \multirow{4}{*}{$\begin{array}{c}\text { Variables } \\
\text { Complementarias }\end{array}$} & Efectividad & 6 & $16 / 24$ \\
\hline & Esfuerzo Extra & 6 & $17 / 24$ \\
\hline & Satisfacción & 5 & $15 / 20$ \\
\hline & Total & 82 & \\
\hline
\end{tabular}




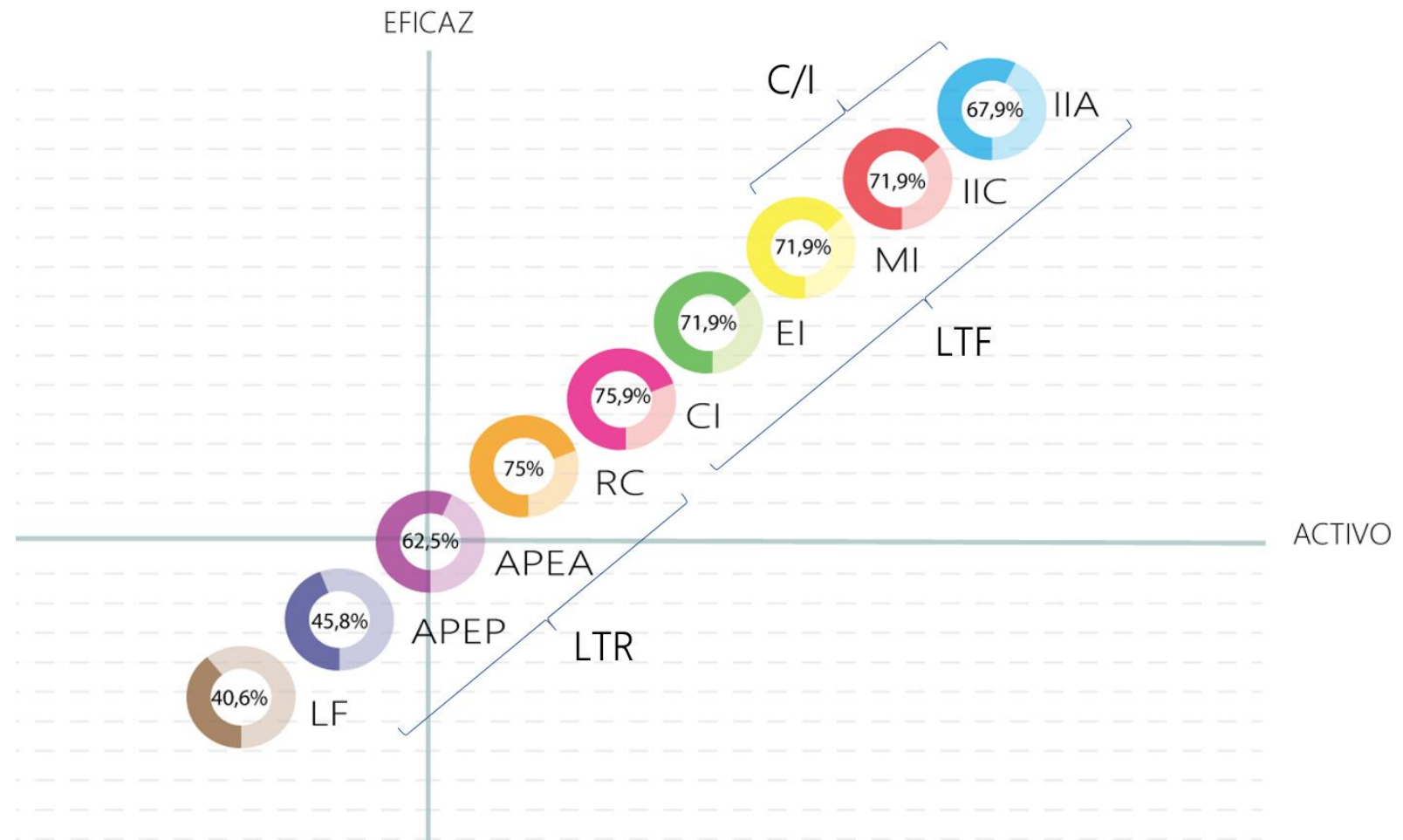

Figura 1: Perfil de Liderazgo del Director de Carrera

Adaptado de (Bass and Avolio, 1994)

LTF: Liderazgo Transformacional

C/I: Carisma/Inspiracional

IIA: Influencia Idealizada Atribuida

IIC: Influencia Idealizada Conducta

MI: Motivación Inspiradora

EI: Estimulación Intelectual

CI: Consideración Individualizada

LTR Liderazgo Transaccional

RC: Reconocimiento Contingente

APEA: Administración Por Excepción Activa

APEP: Administración Por Excepción Pasiva

LF: Laissez-Faire

\section{DISCUSIÓN}

Es indiscutible que los profesionales de ingeniería deben contar con variables de liderazgo destacables, principalmente por las características da gestión, trabajo en equipo y dirección. Dentro del esquema educativo, estas variables se hacen aún más importantes, ya que los esquemas de desarrollo de competencias para los futuros profesionales plantean una necesidad de transferir el tanto competencias blandas como técnicas, para forjar en ellos el perfil profesional que el mercado laboral requiere.

El objeto de este estudio tiene relación con identificar el estilo de liderar, y determinar el estilo aplicado proporciona resultados deseados para la organización. En respuesta a lo anterior, los resultados del análisis obtenido indican que el estilo de liderazgo predominante es el liderazgo transformacional.

El liderazgo transformacional provee de elementos necesario en el proceso formativo de profesionales de Ingeniería Civil Industrial que proporciona un sentido de propósito, con integridad y sentido valórico, estímulo al crecimiento por medio del planteamiento de metas y desafíos, en conjunto con una dinámica de continua retroalimentación.

De manera complementaria, el individuo cuenta con elementos característicos del liderazgo transaccional, indicativos de la labor como Director de Carrera, como el seguimiento de patrones estructurados, mantención de reglas y procesos de toma de decisiones basados en los parámetros de la cultura organizativa, reglamentos, normas y procedimientos establecidos. Aunque la 
mayoría de las dimensiones del liderazgo transaccional reconocidas en el individuo son más activas y cercanas al liderazgo transformacional, entendiendo ambos estilos de liderazgo como complementarios.

Ambos estilos de liderazgo distan cerca del 9\% entre ellas, distancia relativamente pequeña, lo que podría indicar que la complementariedad y balance de ambos estilos puede cumplir un rol fundamental en el escenario de educación superior, cumpliendo con el perfil óptimo de liderazgo. Elementos destacables del análisis son los grados de Efectividad y Satisfacción percibidos por la muestra, alcanzando ambos un $75,0 \%$, lo que sugiere el buen desarrollo del individuo en el cargo analizado. Además, el considerar que el líder logra que sus seguidores estén dispuestos a realizar un Esfuerzo Extra, ítem valorizado en $70,8 \%$, da mayor fortaleza a esta teoría.

Las organizaciones están compuestas por personas, particularmente en las universidades convergen cantidades aún mayores, y cada una de ellas posee realidades de vida distintas, tornando compleja la posibilidad de adoptar un estilo de liderazgo particular. El poder contar estilos de liderazgo complementario, como los liderazgos transformacional y transaccional, permite al individuo a adaptarse al suceso de posibles situaciones, y poder llevar de manera óptima su labor.

Como se indicó, la universidad se encuentra acreditada por 3 años, de un total posible de 7 años, mientras Ingeniería Civil Industrial cuenta con 5 años de acreditación, pudiendo sugerir una relación entre los años de acreditación y la gestión ejercida por los Directores de Carrera. Esta carrera, además de su preocupación por la excelencia en la docencia y el aumento en la investigación, se caracteriza por la realización de variados e importantes proyectos de vinculación y extensión durante su historia, que se ha materializado en actividades como la realización de ferias anuales de innovación, la organización del primer Congreso de Ingeniería Industrial del Norte de Chile INDUNOR 2016, así como el éxito en la participación de concursos de gran envergadura, como la clasificación a semifinal del concurso mundial de simulación de aplicaciones y la carrera Atacama Solar. Actividades que han aumentado sobre todo este último tiempo, por lo que es posible relacionar una incidencia de la labor del director de carrera en el desarrollo de estos proyectos.

Además, se puede considerar elementos como el haber ganado el concurso de electromovilidad y la participación y selección de alumnos para la Carrera Atacama Solar y el concurso AULAB, como elementos característicos de la gestión de este director de carrera, así como el incremento en la participación en actividades de extensión y vinculación con el medio, como factores claves de su estilo de liderazgo.

Estudios previos desarrollados en organizaciones exitosas en distintos rubros distribuidas por varios países de Sudamérica poseían gerentes y altos directivos que contaban con liderazgos de tipo transformacional de manera predominante (Martínez-Quezada, 2010, Martínez-Quezada et al., 2013, Martínez-Quezada et al., 2014), situación que concuerda con los resultados obtenidos en esta investigación y el estilo predominante reconocido en el Director de carrera. Además, considerando las actividades y reconocimientos conseguidos por su gestión, se puede establecer un vínculo entre su estilo de liderazgo y logros obtenidos. Por ello se sugiere iniciar otra línea de investigación, para determinar si el factor de liderazgo incide en los años de acreditación, de las diferentes carreras de la Universidad Arturo Prat.

\section{AGRADECIMIENTOS}

El presente trabajo va dedicado con mucha gratitud a Raúl Castillo Villagra y a la Facultad de Ingeniería y Arquitectura de la Universidad Arturo Prat, quienes fueron un aporte fundamental para la realización de esta investigación, expresados por su disposición y compromiso para el desarrollo de esta.

\section{REFERENCIAS BIBILOGRÁFICAS}

Avolio, B. J. y Bass, B. M. (1988) Transformational leadership, charisma, and beyond. In: HUNT, J. G., BALIGA, B. R., DACHLER, H. P. \& SCHRIESHEIM, C. A. (eds.) Emerging leadership vistas. Lexington, $\mathrm{MA}$, England: Lexington Books/D. C. Heath and Com. 
Avolio, B. J., Bass, B. M. y Jung, D. I. (1999) Re-examining the components of transformational and transactional leadership using the Multifactor Leadership. Journal of Occupational and Organizational Psychology, 72, 441-462.

Bas, B. M. (1985) Leadership and performance beyond expectations, Free Press; Collier Macmillan.

Bass, B. M. (1995) Theory of transformational leadership redux. The Leadership Quarterly, 6, 463-478.

Bass, B. M. (1997) Does the transactionaltransformational leadership paradigm transcend organizational and national boundaries? American Psychologist, 52, 130-139.

Bass, B. M. (1998) Transformational Leadership: Industry, Military and Educational Impact. Lawrence Erlbaum Associates.

Bass, B. M. y Avolio, B. J. (1993) Transformational leadership and organizational culture. Public administration quarterly, 17 (3-4), 112121.

Bass, B. M. y Avolio, B. J. (1994) Improving Organizational Effectiveness through trasformational leadership, SAGE Publications, Inc.

Burns, J. M. (1978) Leadership, New York, Harper \& Row.

CNA-CHILE, (Comisión Nacional de Acreditación). (2016) Buscador de Acreditaciones. Disponible en: https://www.cnachile.cl/ [Consulta: 04 febrero 2016].

Den Hartog, D. N., Van Muijen, J. J. y Koopman, P. L. (1997) Transactional versus transformational leadership: An analysis of the MLQ. Journal of Occupational and Organizational Psychology, 70, 19-34.

Eagly, A. H. y Johannesen-Schmidt, M. C. (2007). "Leadership style matters: the small, but important, style differences between male and female leaders". En: Bilimoria, D. y Piderit, S.K. eds. Handbook on women in business and management [en línea] Disponible en:

http://www.elgaronline.com/view/978184 5424329.00023.xml [Conculta: 03 marzo 2006].
Hater, J. J. y Bass, B. M. (1988) Superiors' evaluations and subordinates' perceptions of transformational and transactional leadership. Journal of Applied Psychology, 73, 695-702.

Horn, A. y Marfán, J. (2010) Relación Entre Liderazgo Educativo y Desempeño Escolar: Revisión de la Investigación en Chile. Psicoperspectivas, 9, 82-104.

Judge, T. A. y Bono, J. E. (2000) Five-factor model of personality and transformational leadership. Journal of Applied Psychology, 85, 751-765.

Judge, T. A. y Piccolo, R. F. (2004) Transformational and Transactional Leadership: A Meta-Analytic Test of Their Relative Validity. Journal of Applied Psychology, 89, 755-768.

Lowe, K. B., Kroeck, K. G. y Sivasubramanian, N. (1996) Effectiveness correlates of transformational and transactional leadership: A meta-analytic review of the mlq literature. The Leadership Quarterly, 7, 385-425.

Martínez-Quezada, A. (2010) "Propuesta de investigación del liderazgo como variable determinante del resultado de las pymes", Perspectiva, 11 (13), pp. 37-45.

Martínez-Quezada, A., Orrego-Saavedra, G. y Contreras-Alfaro, A. (2014) Identificación del estilo de liderazgo en una empresa (cooperativa) de servicios sanitarios. Perspectiva, 15 (17), 52-63.

Martínez-Quezada, A., Valdenedro-Avendaño, A., González-Harvey, D. y Rodríguez Álvarez, H. (2013) Identificación del estilo de liderazgo en una empresa de hidrocarburos. Perspectiva, 14 (16), 5969.

Riaga, C. O. y Torres, M. R. M. (2006) El liderazgo transformacional, dimensiones e impacto en la cultura organizacional y eficacia de las empresas. Revista Facultad de Ciencias Económicas: Investigación y Reflexión, 14, 118-134.

Rubino, A. N. (2007) Desafios de la gerencia y el liderazgo de la educación superior. Investigación y Postgrado, 22 (2), 147163. 
Thieme, C. (2005) Liderazgo y eficacia en la educación primaria. El caso de Chile. [en línea] Ingeniería Civil Industrial. Disponible en:

http://www.unap.cl/admision/2016/psu/2 3012/index.html [Consultado 08 enero 2016].
Vega-Villa, C. y Zavala-Villalón, G. (2004) Adaptación del Cuestionario Multifactorial de Liderazgo (MLQ Forma $5 X$ Corta) de $B$. Bass y $B$. Avolio al contexto organizacional chileno., Universidad de Chile. 